\title{
Wie Apps marode Körper heilen
}

\author{
Teletherapie und Software-Applikationen spielen in der Physiotherapie eine \\ zunehmende Rolle. Nur die Krankenkassen üben sich in großer Zurückhaltung. \\ Von Stephanie Dirnbacher
}

Stoppuhr, Papier und Stift braucht Simon Kanzian für den sechsminütigen Gehtest nicht mehr. Auch die Distanz, die die Patienten beim Test zurücklegen, muss er nicht mehr messen. Stattdessen schnallt der Physiotherapeut im Orthopädischen Spital Speising (OSS) den Patienten einen Monitor in der Größe eines Smartphones um die Hüfte und wartet auf die Auswertung der Bewegungsdaten. Sie erzählen ihm von der Ausdauer des Patienten.

\section{Auf die Beine bringen}

Seit Oktober 2020 testet das Spital Speising die als Medizinprodukt zertifizierte Lösung des Wiener Start-ups Reha Buddy an allen ambulanten Rehapatienten. „Die digitalisierte Messung erleichtert die Dokumentation, weil die Daten direkt vom Monitor erhoben und ausgewertet werden. Das Testen und die Befundung gehen dadurch schneller und wir können schneller mit der Therapie beginnen“, schildert Kanzian den digitalen Arbeitstag. Und es gibt noch einen Vorteil: „Der Fehlfaktor Mensch fällt weg. Die Datenerfassung ist genauer und objektiver." Der digitale Test liefert darüber hinaus zusätzliche Informationen, etwa über die Gehgeschwindigkeit im Zeitverlauf oder über die Symmetrie des Ganges. Harald Jagos, CEO und Mitbegründer von Reha Buddy, sieht einen weiteren Einsatzbereich bei Long-Covid-Patienten. Neben dem 6-Minuten Gehtest entwickelt Reha Buddy noch weitere digitale Assessments für die Physiotherapie. Der digitale Timed-Up-And-Go-Test wird demnächst in den Wiener Pensionisten-Wohnhäusern zur Mobilisierung von geriatrischen Patienten eingesetzt. Die Applikation des Sitto-Stand-Tests soll bis Ende des Jahres fertig werden. Momentan befindet sich das Start-up in der Phase des Markteintritts.

Reha Buddy ist eine von vielen Lösungen, die darauf abzielen, die Physiotherapie zu digitalisieren. „Wir merken, dass digitale Lösungen stark im Kommen sind. Immer mehr Firmen bieten uns digitale Produkte zur Unterstützung an. Das Spital Speising legt den Schwerpunkt darauf, die Befundung zu digitalisieren und papierlos zu werden“, erzählt Kanzian.

\section{Bessere Datenauswertung}

Constance Schlegl, Präsidentin von Physio Austria, dem Bundesverband der Physiotherapeuten in Österreich, sieht mehrere Vorteile von digitalen Applikationen: „Einerseits erleichtern sie die Datenauswertung und die Dokumentation über die Entwicklung der Patienten. Das führt zu einer Zeitersparnis. Andererseits ist der Austausch von Informationen zwischen berechtigten Berufsgruppen einfacher. In einigen Fällen verbessert die Digitalisierung auch die Adhärenz, also das Ausmaß, zu dem das Verhalten der Patienten mit den Therapieempfehlungen übereinstimmt. Vor allem technikaffine Menschen lassen sich mit digitalen Tools in der Therapie oft besser motivieren." 
Schlegl weiß aber auch um die Probleme und Herausforderungen beim Einsatz von digitalen Lösungen: „Es ist wichtig, immer die grundlegenden ethischen Prinzipien der Physiotherapie einzuhalten, wie etwa den 1:1-Kontakt. Unethisch wäre es auch, eine App zu empfehlen, ohne vorher abzuklären, ob der Patient dafür geeignet ist." Tatsächlich ist nicht jeder Patient vom digitalen Fortschritt begeistert. Vielleicht hat er Probleme beim Ausführen der Übungen oder es sind manche Übungen für ihn kontraproduktiv. Genauso wichtig ist die Datensicherheit. Wenn eine App die Daten auf einem Server in einem Land speichert, das keinen ausreichenden Datenschutz gewährleistet, darf man sie nicht empfehlen. Es darf auch keine Vorratsdatenspeicherung erfolgen. „Bei digitalen Gesundheitslösungen ist die Angst vor dem gläsernen Patienten sehr groß“, erklärt Schlegl.

Datensicherheit ist also ein Knackpunkt. Da bei den physiotherapeutischen Applikationen in der Regel hochsensible Daten gespeichert und verarbeitet werden, müssen die Lösungen sehr strenge Auflagen erfüllen. „Die Daten dürfen den Patienten nur in der Klinik zuordenbar sein. Auch bei der Speicherung müs-

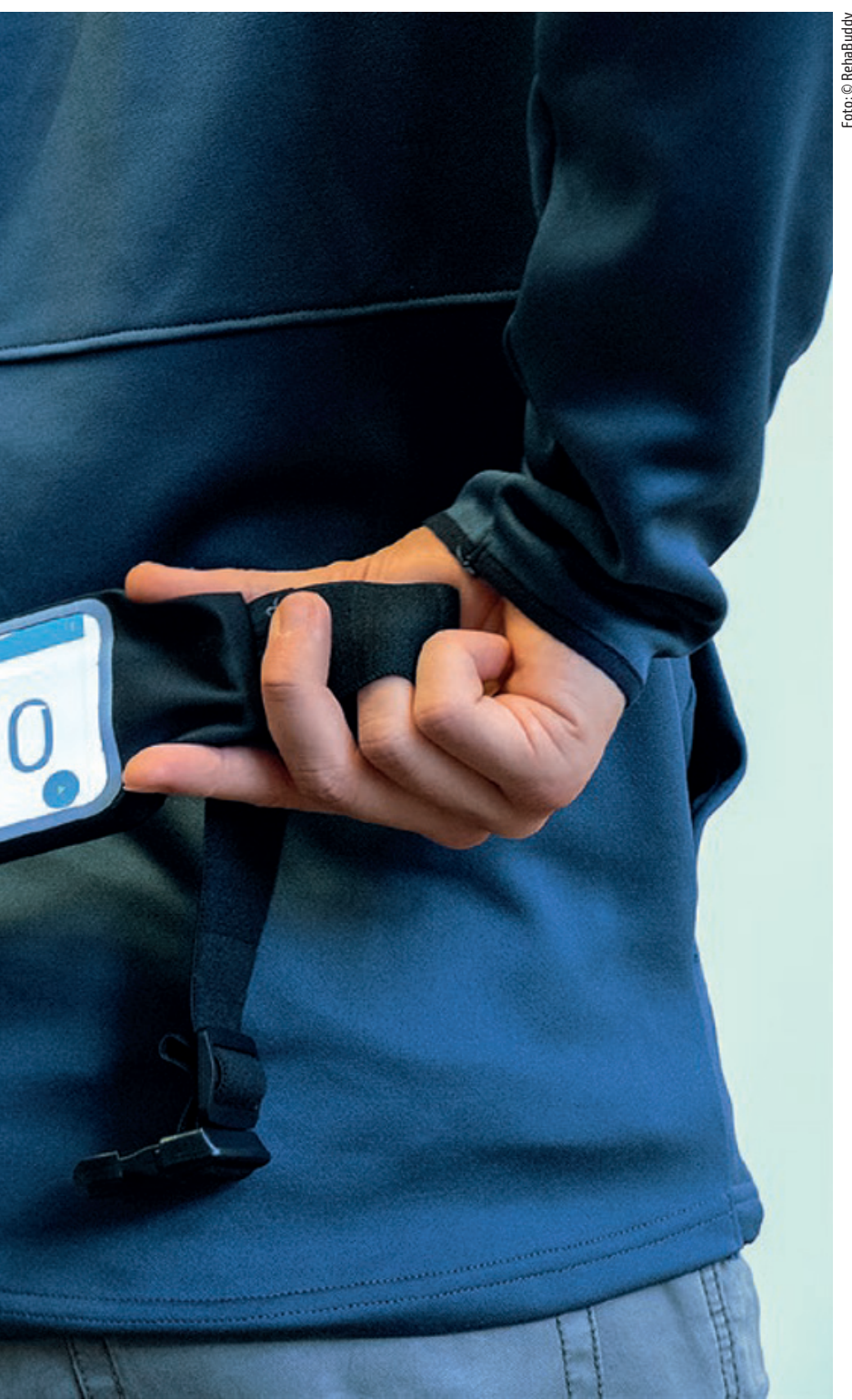

sen hohe Sicherheitsstandards eingehalten werden", weiß RehaBuddy-CEO Jagos. Bei Reha Buddy werden die Patientendaten bislang nur lokal auf den Geräten gespeichert. Geplant ist eine Anbindung an das Krankenhausinformationssystem.

\section{Teletherapie auf dem Vormarsch}

Die Digitalisierung hält in die Physiotherapie nicht nur über Apps Einzug. Mit Covid-19 ist auch die Teletherapie salonfähig geworden. Teletherapie ist ein breit umfassender Begriff und meint sowohl Online-Konsultationen als auch den Einsatz von Systemen, die dem Patienten mithilfe sensorischer Apparaturen eine Interaktion mit Geräten oder dem online agierenden Therapeuten ermöglichen.

Voraussetzung für alle Formen der Teletherapie ist die sichere Übertragung medizinischer Daten für Prävention, Diagnose, Behandlung und Weiterbetreuung. Die Formate sind dabei unterschiedlich: Die Kommunikation kann über Text, Ton und/oder Bild stattfinden. Hauptanwendungsgebiete der Teletherapie, als telemedizinischer Dienst zwischen Patienten und Gesundheitsdienstanbieter, sind chronische Erkrankungen wie Herz-Kreislaufkrankheiten und Diabetes. Covid hat - wie in allen digitalen Bereichen - die Entwicklung stark beschleunigt: Laut einem Briefing Paper des Weltverbands der Physiotherapie über die Entwicklung digitaler Versorgungsformen hat sich die Anwendung von Teletherapie während der Pandemie bei älteren Patienten, im Bereich Frauengesundheit und bei kardiopulmonalen Indikationen jeweils verdoppelt. Niedergelassene Physiotherapeuten verzeichneten im pädiatrischen Bereich einen Anstieg teletherapeutischer Maßnahmen um 450 Prozent.

Physio-Austria-Präsidentin Schlegl ist sich sicher, dass Teletherapie auch nach der Pandemie eine Versorgungsform in der Physiotherapie bleiben wird. „Wir arbeiten gerade mit der Österreichischen Gesundheitskasse an einem bundesweiten Rahmenvertrag, der die Kostenerstattung regelt und Teletherapie als eigene Leistung etabliert", verrät sie.

Die Basis ist gelegt: In Österreich ist Telerehabilitation gesetzlich bereits seit 2018 als medizinische Maßnahme der Rehabilitation verankert. Doch die Verrechnung mit der Krankenkasse ist immer nur unter bestimmten Voraussetzungen möglich. Die Pan-

\section{DER NUTZEN VON TELETHERAPIE}

Ein Bericht des Weltverbands für Physiotherapie und des Internationalen Netzwerks der Regulierungsbehörden für Physiotherapie (INPTRA) listet folgende Vorteile der digitalen Behandlung für Patienten auf:

- Bessere Zugänglichkeit zur Physiotherapie, insbesondere in ländlichen Regionen mit großen Distanzen zu den physiotherapeutischen Praxen

- Wegfall von Barrieren wie Wegzeiten oder Parkplatzsuche

- Mehr Patientensicherheit für immobile und kranke Patienten, bei denen sich eine Fahrt beziehungsweise ein Transport in die Praxis negativ auf den Gesundheitszustand auswirken kann

- Mehr zeitliche Flexibilität

- Kostenersparnis in Hinblick auf Fahrtkosten oder Unterstützungsleistungen, die durch die Abwesenheit des Patienten von zu Hause nötig werden, wie zum Beispiel Kosten für einen Babysitter. 


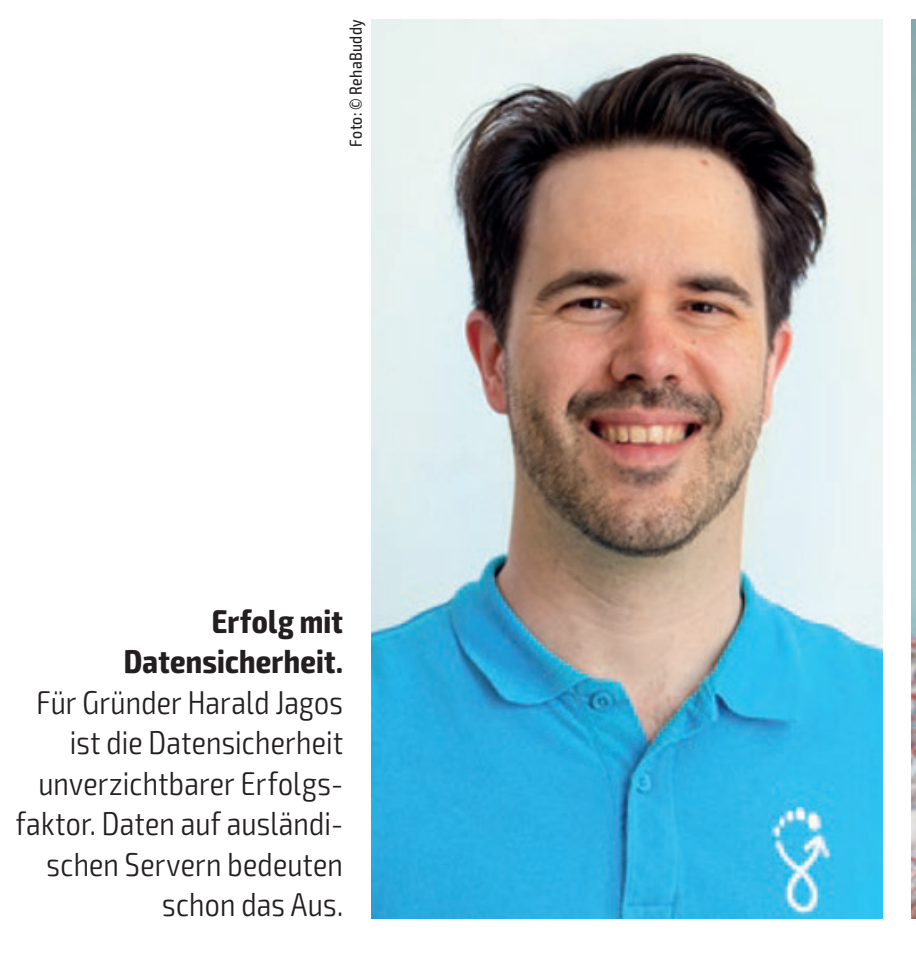

demie hat hier als echter Game Changer gewirkt. „Mit Covid-19 hat die Kostenerstattung von teletherapeutischen Behandlungen sehr rasch funktioniert“, erzählt Schlegl.

"Auch zukünftig soll die telemedizinische Behandlung in Ausnahmefällen, wenn persönliche Behandlungen nicht möglich sind, unter bestimmten Voraussetzungen durchgeführt werden können", heißt es dazu von der Österreichischen Gesundheitskasse (ÖGK). Als Voraussetzungen führt die ÖGK unter anderem die Zustimmung der Patienten zur Teletherapie, die Wahrung des Patientenwohls, ein geeignetes technisches Equipment sowie die Einhaltung des Datenschutzes an. Auch Schlegl bevorzugt den direkten Kontakt zwischen Physiotherapeuten und Patienten. „Wir sehen Teletherapie als Ressource und Ergänzung zur traditionellen Physiotherapie. Sie soll dort zum Einsatz kommen, wo sie einen Nutzen für Patienten hat, wenn sie zum Beispiel den Zugang zu den Behandlungsangeboten verbessert und/oder die Therapie effektiver macht.“

\section{Technische Infrastruktur als Herausforderung}

Dem breiten Einsatz von Teletherapie in der Physiotherapie stehen jedoch einige Hindernisse entgegen. Neben Fragen zur Kostenerstattung sind vor allem unklare Regulierungen, fehlende Möglichkeiten von Hands-on-Assessments und -Interventionen sowie Herausforderungen im Zusammenhang mit der technischen Infrastruktur - sowohl auf Seite der behandelnden Therapeuten als auch auf Seite der Patienten - zu nennen. Dieser letzte Punkt wird auch in dem Briefing Paper des Weltverbands der Physiotherapie als größte Barriere angeführt. „Man darf sich das nicht so vorstellen, dass der Physiotherapeut den Patienten über Skype behandelt. Seriöse Teletherapie beruht auf einer adäquaten datenschutzsicheren Software-Lösung. Je nach Methodenwahl ist neben der normalen Webcam auch eine zusätzliche Sensorik und leistungsstarkes, störungsfreies Internet sowohl beim Patienten als auch beim Therapeuten erforderlich“, führt Schlegl dazu aus.

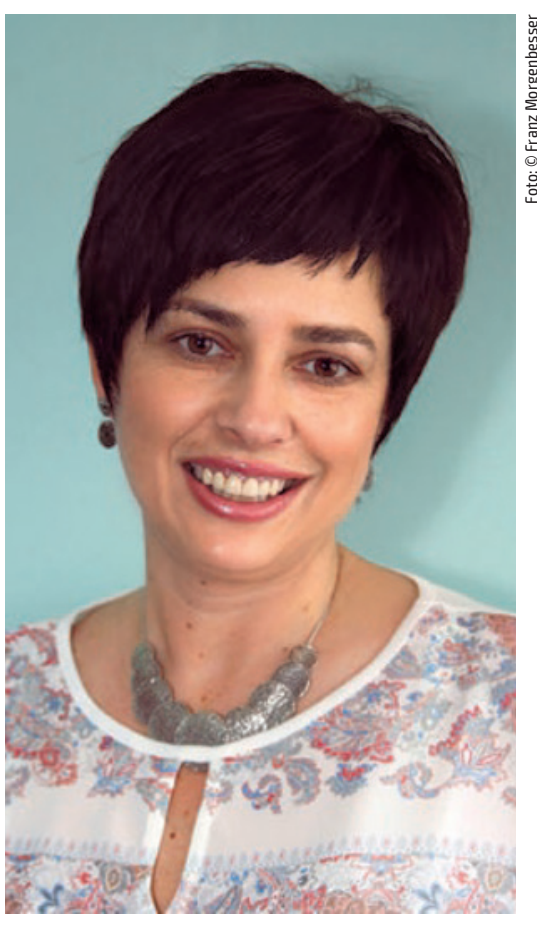

Spielerische Motivation.

Für Constance Schlegl, Präsidentin von Physio Austria, lassen sich technikaffine Menschen mit digitalen Tools in der Therapie besser motivieren. Mit "Serious Games" werden unerwartete Erfolge erzielt.
Aktuell gibt es noch nicht viele funktionierende Softwarelösungen, die den strengen Bestimmungen über Datenschutz und Patientenrechte in Österreich genügen. Die Kosten für eine Software und entsprechende Hardware bewegen sich teilweise im vierstelligen Bereich.

\section{Positive Einstellung von Patienten}

Die Akzeptanz von digitaler Physiotherapie durch die Patienten wird von medizinischer Seite stark hinterfragt - ohne auf große Unterschiede zu stoßen. Eine amerikanische Studie (Eannucci EF, et al.: Patient Satisfaction for Telehealth Physical Therapy Services ..., National Library of Medicine Pubmed.gov, 2020) verglich die Patientenzufriedenheit bei persönlicher Physiotherapie mit jener bei digitaler Behandlung. Die Studienautoren konnten keine bedeutenden Unterschiede in der Zufriedenheit feststellen. Eine andere, ebenfalls amerikanische Studie (Miller MJ, Pak SS, et al.: Evaluation of Pragmatic Telehealth Physical Therapy Implementation During the COVID-19 Pandemic, National Library of Medicine Pubmed.gov, 2021) untersuchte die Zufriedenheit der Patienten anhand von 4548 Behandlungseinheiten, von denen 85 Prozent digital abgewickelt wurden. Die Zufriedenheit der befragten Probanden war mit 94 Prozent hoch; 92 Prozent waren bereit, wieder an einer Telerehabilitation teilzunehmen.

\section{Neuausrichtung der Ausbildung}

Zur Etablierung der Teletherapie in der physiotherapeutischen Praxis braucht es jedenfalls Klarheit bezüglich der rechtlichen Rahmenbedingungen, der Kostenerstattung und der Qualitätsanforderungen, so Schlegl. Der Bundesverband der Physiotherapeuten will bis Jahresende Qualitätsrichtlinien für Teletherapie erarbeiten. Schlegl hält es auch für wichtig, die Digitalisierung in die Ausbildung von Physiotherapeuten zu integrieren. „Derzeit gibt es zu dem Thema in Österreich nur Weiterbildungsoptionen“, sagt sie. 